\title{
Efficient Search of Winning Strategies in Multi-agent Systems on Random Network: Importance of Local Solidarity
}

\author{
Tin Yau Pang and K.Y. Szeto \\ Department of Physics, \\ Hong Kong University of Science and Technology, \\ Hong Kong \\ phszeto@ust.hk
}

\begin{abstract}
Multi-agent systems defined on a network can be used for modelling the competition between companies in terms of market dominance. In view of the enormous size of the search space for winning strategies of initial configuration of resource allocation on network, we focus our search on the subspace defined by special local clustering effects, using the recently developed evolutionary computational algorithm. Strategies that emphasize local solidarity, measured by the formation of clusters in the form of triangles linkage between members of the same company, prove to be effective in winning both the market share with high probability and high speed. The result provides a good guideline to improve the collective competitiveness in a network of agents. The formulation is based on the Ising model in statistical physics and the evolutionary game is based on Monte Carlo simulation. Significance and the application of the algorithm in the context of econophysics and damage spreading in network are discussed.
\end{abstract}

\section{Introduction}

The resource allocation problem is an interesting problem in econophysics where it considers different competitors on the market trying to achieve market dominance in the shortest time [1]. A good example can be found in the competition between telecommunication service providers on a social network. The telecommunication service providers are interested in expanding their business in terms of subscribing clients by good allocation of their resource. It is a common promotional practice that the service providers offer a lower price or other bonus to reward the clients to make intra-network phone calls, that is, phone call made between users subscribing the same company. In this way, clustering in social networks becoming clustering of the clients for the company. Users will find the promotional offer of intra-network call attractive, as most calls will be made between friends. We therefore anticipate that clustering effects will be a useful guideline in the design of winning strategy in resource allocation.

If one maps the clustering effect of social networks to the problem of local interactions in econophysics, one natural description will be the localized magnetic moment models commonly studied in statistical physics. A simplest model for two 
competing companies is the Ising model on the network. The model consists of a set of Ising spins with two possible values, up or down, (representing two companies, say, black or white). The local interaction with neighbouring spins will determine the spin orientation probabilistically, according to the Boltzmann factor. In the ferromagnetic version of the Ising model, if most of the spin in the neighbouring sites are pointing up (down), then it is likely to point up (down). In order to relate this model to the problem of the resource allocation for the telecommunication service provider, we assume that the clients are living in an artificial society defined by a social network [2] where every node of the network represents a client and a pair of connected nodes means that the pair of clients communicates frequently on the phone. (Here we consider a bi-directional network). We approach the problem with the Ising model of statistical mechanics in physics [3] and model the social network with a random network [4]. Every node of the network has an Ising spin [5], with a freedom to choose its state (up or down), corresponding to the service provider subscribed. The approach to the resource allocation problem is: (1) generate a set of initial spin configuration on the network, (2) simulate the initial configurations using Monte Carlo simulation [6] to find the probability and speed for the different telecommunication service provider to achieve dominance on the network and (3) evolve the initial spin configuration with Mutation Only Genetic Algorithm (MOGA) [7,8,9] to improve the winning probability and speed of dominance of the spin configuration. Here speed is the inverse of time required for a service provider to achieve dominance, which is a good parameter to assess the competence of a strategy.

\section{Modelling of the Resource Allocation Problem}

The resource allocation problem of the telecommunication service providers can be modelled by multi-agent system defined on a random network simulated by Monte Carlo simulation [1]. A random network with $L$ nodes with connection probability $p$ is formed by linking every pair of nodes with probability $p$. Every node on the network represents a client in the model, and a link between two nodes represents their established communication. Here we assume that only two telecommunication service providers are competing in order to simplify the model. For a node $i$ there is a spin $s_{i}$ residing on it. The state of spin (up: $s_{i}=+1$ or down: $s_{i}=-1$ ) represents the subscribed telecommunication service provider. The interaction energy $E$ between two spins is defined as

$$
E=-\sum_{i>j}^{L} J_{i j} s_{i} s_{j}
$$

Here $J_{i j}$ is 1 if node $i$ and $j$ are connected in the network and 0 otherwise. The energy $E$ is raised by a pair of nodes with opposite spin type and lowered otherwise. If we interpret $E$ as some monotonic increasing function of the total spending of the client on the telecommunication service, then we expect that $E$ tends to drop throughout the simulation since people are eager to minimize their cost. To make a fair game, we assume that none of the service providers has enough resource to supersede its competitors so that we constrain the initial configuration of the model to be any spin 
configuration with equal dominance of different spin types: the two service providers have $50 \%$ market share at the beginning. In our simulation we studied two random network both having $L=60$, one with $p=0.05$ and the other with $p=0.1$.

\section{Monte Carlo Simulation to Evolve the Initial Spin Configuration on the Random Network}

The initial spin configurations in the model are evolved by Monte Carlo simulation. In every step of the Monte Carlo simulation a spin $s_{i}$ is chosen from the network. Let $\Delta E$ be the energy change associated with the switching of $s_{i}$, the probability to switch $s_{i}$ in the Monte Carlo step is 1 if $\Delta E \leqq 0$. If $\Delta E>0$ then it is switched with probability $P$ :

$$
P=\exp \left(-\frac{\Delta E}{k T}\right)
$$

where $k$ is the Boltzmann constant and $T$ is the temperature of the network. The presence of a temperature in the equation is related to the noise in the competition that affects the decision of a client. Government policies and various advertisements or promotions are examples of the noise [1]. We set $k T=1$ in our simulation. This is a temperature sufficiently low to guarantee that one of the two spin type will be able to dominate the network. If $k T$ is too high then none of the spin types will be able to dominate the network. (In the language of the physics of magnetism, the high temperature phase is paramagnetic, without ferromagnetic order [5].) This is an undesirable condition since we are not able tell which one has achieved dominance and we cannot make any conclusion.

In the Monte Carlo simulation the steps are carried on until one of the spin types has achieved dominance. To save computational resource we do not wait for a spin type to dominate the whole network. Instead we declare dominance of one spin type once it has occupied $87.5 \%$ of the network. Furthermore it is possible but rarely occurs that the simulation will enter a deadlock where none of the spin type is able to defeat the other and achieve dominance. When such cases occur we declare a draw. In our simulation, we define a draw if no winner appears after 100,000 Monte Carlo steps. This is a rare situation and we do not count such cases in our analysis.

\section{Search of Winning Configurations by Genetic Algorithm}

On an $L=60$ random network, there are ${ }_{60} \mathrm{C}_{30}\left(1.2 \times 10^{17}\right)$ valid initial spin configurations. To handle the search for winning initial configuration in such an enormous search space, we applied the recently developed Mutation Only Genetic Algorithm (MOGA) [7,8,9] which we found to be very efficient in solving the Knapsack problem. We first map the spin configuration bijectively on a chromosome containing 60 elements, where each element could be 0 or 1 . Let the entire population of $N$ chromosome each having a length $L(=60)$ expressed as a matrix $A_{i j}(t), i=1, \ldots, N$; $j=1, \ldots, L$, where $A$ has been sorted according to the fitness of chromosome such that 
the $i^{\text {th }}$ chromosome has a fitness equal or higher than the $i+1^{\text {th }}$ chromosome. We consider the mutation matrix $M(t)$ such that $M_{i j}(t)=a_{i}(t) b_{j}(t), i=1, \ldots, N ; j=1, \ldots, L$, where $0 \leqq a_{i}(t), \quad b_{j}(t) \leqq 1$ are respectively called the row (column) mutation probability. We should mutate loci on an unfit chromosome and we set

$$
a_{i}(t)=1-\left(\frac{\sum_{n=i}^{N} f(n)}{\sum_{n=1}^{N} f(n)}\right)
$$

where $f(n)$ is the fitness of the chromosome $n$ and $a_{i}(t)$ is zero for the fittest chromosome in the population and largest for the weakest chromosome. We then consider the column mutation probability $b_{j}(t)$. Let's define the locus mutation probability of changing to $X(X=0$ or 1$)$ at locus $j$ as $p_{j X}$ :

$$
p_{j X}=\frac{1}{\sum_{m=1}^{N} m}\left(\sum_{k=1}^{N}(N+1-k) \delta_{k j}(X)\right)
$$

where $\delta_{k j}(X)$ is 1 if the $j^{\text {th }}$ element of the $i^{\text {th }}$ chromosome has locus $X$, and 0 otherwise. Note that $p_{j X}$ contains information of both locus and row, and the locus statistics is biased so that heavier weight for chromosomes with high fitness is assumed. After defining $p_{j X}$ we define the $b_{j}(t)$ as:

$$
b_{j}=\frac{1}{\sum_{j=1}^{L} b_{j}}\left(1-\left|p_{j 0}-0.5\right|-\left|p_{j 1}-0.5\right|\right)
$$

Let's consider an example. If 0 and 1 are randomly distributed then $p_{j 0}=p_{j l}=0.5$, we have no useful information about the locus and we set $b_{j}=1$. When there is definitive information, such as when $p_{j 0}=1-p_{j l}=0$ or 1 , we should not mutate and $b_{j}(t)=0$. As there are sixty nodes in the network and the spins in every node can take two values, we can represent all configurations by a chromosome which is a sequence having thirty $1 \mathrm{~s}$ and thirty 0s. To compare different strategies for the initial allocation of resource, we define a fitness for the spin configuration. The evolution of initial spin configurations affects both spin types. If the evolution favours one spin type and make it more competent then at the same time it will reduce the competitiveness of the other spin types. Here we set the evolution process to favour the spin up type and so the fitness of a chromosome solely reflects the competitiveness of the spin up type. Given a chromosome representing a particular initial spin configuration, we perform $U$ trials of Monte Carlo simulation. If there are $u$ out of the $U$ trials of Monte Carlo simulations spin up achieve $87.5 \%$ dominance, then $U-u$ times spin down will win. We now define for a given trial the number $s$ as the metropolis steps required to evolve the initial configuration to one that has $87.5 \%$ dominance by one spin type, say 
the spin up type. Therefore, among the $U$ trials, we see that the average speed to dominance by spin up is given by $\langle 1 / s\rangle_{u}$, while the average speed to dominance by spin down is given by $\langle 1 / s\rangle_{U-u}$. Other than the speed and probability for spin up type to achieve dominate, we are interested in the number of triangles formed by the two spin types. Here a triangle is defined as a group of three interconnected nodes that share the same spin type, and we denote $T^{U}$ and $T^{D}$ the number of triangles of spin up type and spin down type. We expect that the number of triangle is a good indicator for solidarity of the two spin types: the larger the different of number of triangle between the two spin the larger the disparity in terms of solidarity. Thus the term $T^{U}-T^{D}$ should be included in our consideration of fitness of the chromosome. Furthermore, we normalize this factor by dividing $\left(T^{U}-T^{D}\right)$ by $\left(T^{U}+T^{D}\right)$, and add a constant factor 1 so that the fitness is non-negative. This inclusion of the effect of local solidarity, represented by the factor $\left\{1+\left(T^{U}-T^{D}\right) /\left(T^{U}+T^{D}\right)\right\}$ into the fitness definition allows us to direct our search to the subspace with concentrated clustering effects. We now define the fitness $f_{c}$ of chromosome $c$ by the average speed to dominance by spin up, multiplied by the probability that spin up dominates among $U(=1000)$ trials,

$$
f_{c}=\left\langle\frac{1}{s}\right\rangle_{u} \times\left(\frac{u}{U}\right) \times\left[1+\frac{T^{U}-T^{D}}{T^{U}+T^{D}}\right]
$$

Since we want to compare the strategies of two companies with initially equal market share, we must make sure that in the construction of initial spin configurations there are equal number of spin up and spin down. However, the mutation operation in genetic algorithm will not conserve the number of spin up and down (represented by 1 and 0 ). We thus need to modify MOGA. Let's assume that the mutation probability for the locus in the chromosome has been calculated in the usual way [8], and that the chromosome $\mathrm{c}$ has $k$ loci to be mutated. Out of these $k$ loci $k_{0}$ of them are $0 \mathrm{~s}$ and $k_{l}$ of them are $1 \mathrm{~s}$. We only mutate $2 \times \min \left(k_{0}, k_{1}\right)$, and reject those 0 s or $1 \mathrm{~s}$ in excess which have the least mutation probability in order to conserve the number of $0 \mathrm{~s}$ and $1 \mathrm{~s}$. For example, MOGA has designated the first six loci of the chromosome 1111000010 to mutate, and the corresponding mutation probabilities are: $0.90 .70 .5 \underline{0.3} \underline{0.8} 0.60 .2$ 0.10 .050 .04 respectively. To conserve the number of $0 \mathrm{~s}$ and $1 \mathrm{~s}$, two $1 \mathrm{~s}$ should not mutate. Since the third and the fourth 1 are the least probable to mutate among all chosen 1s, they won't mutate. So far the final outcome is 0011110010. In our evolutionary computation using MOGA, we set the population size of chromosome to be $100(=\mathrm{N})$ and the number of generation for the chromosomes to evolve to be 100 .

\section{Result of the Simulation}

We performed simulations on two random networks which have $N=60$ nodes, one with $p=0.05$ and the other $p=0.1$. For each random network we performed evolution with genetic algorithm described in Section 4, with the fitness evaluation defined by the Monte Carlo process in Section 3, and output the chromosome with the highest fitness. We repeat this 10 times with different initial population and random number seed, so as to obtain 10 best chromosomes for the random network, corresponding to 10 best initial spin configurations. We measured $\langle u / U\rangle,\langle 1 / s\rangle_{u}$ and $\left\{1+\left(T^{U}\right.\right.$. $\left.\left.T^{D}\right) /\left(T^{U}+T^{D}\right)\right\}$ on these 10 initial spin configurations, as well as their distributions of 
connectivity of nodes and the clustering coefficient. Here the connectivity of node $i$, denoted as $k_{i} \equiv C_{i}^{S}+C_{i}^{D}$, stands for the number $\left(C_{i}^{S}\right)$ of the neighbours having the same spin type as $i$ 's plus the number of the neighbors $\left(C_{i}^{D}\right)$ having opposite spin types as node $i$. Let's denote $\mathbf{U}$ and $\mathbf{D}$ to be the set that contains nodes with spin up type and spin down type respectively, the distribution of spin up type (the favoured spin type) and spin down type (the hampered spin type) are defined as the distribution of elements in the set $\left\{k_{i}, i \in U\right\}$ and $\left\{k_{i}, i \in D\right\}$. Next denote $T_{i}$ as the number of triangles having a node being $i$, and let's introduce a local measure $d_{i}$ on node $i$

$$
d_{i}=\left\{\begin{array}{ccc}
0 & \text { if } & k_{i}<2 \\
\frac{T_{i}}{k_{i}\left(k_{i}-1\right) / 2} & \text { if } & k_{i} \geq 2
\end{array}\right.
$$

The clustering coefficient of the spin up type and spin down type is defined as the average $\left\langle d_{i}\right\rangle\{i \in U, D\}$. Statistics shows (i) the enhanced strategies have a higher probability to achieve dominance on the network, (ii) the favoured spin have more triangles than its counterpart (Table 1). This is very natural if we interpret the number of triangle as the indicator of solidarity: the more united it is the harder to break it.

Table 1. Averaged $\langle 1 / s\rangle_{u}, u / U$ and $1+\left(T^{U}-T^{D}\right) /\left(T^{U}+T^{D}\right)$ for 10 random spin configurations and 10 good spin configurations on the two networks

\begin{tabular}{|c|c|c|c|c|c|}
\hline \multicolumn{6}{|c|}{$P=0.05$} \\
\hline \multicolumn{3}{|c|}{ Random Spin Configuration } & \multicolumn{3}{|c|}{ Good Spin Configuration } \\
\hline$<1 / s>_{u}$ & $u / U$ & $1+\frac{T^{U}-T^{D}}{T^{U}+T^{D}}$ & $<1 / s>_{u}$ & $u / U$ & $1+\frac{T^{U}-T^{D}}{T^{U}+T^{D}}$ \\
\hline $0.0023 \pm 0.0002$ & $0.47 \pm 0.22$ & $1.0 \pm 1.0$ & $0.0036 \pm 0.0003$ & $0.93 \pm 0.05$ & $1.8 \pm 0.6$ \\
\hline \multicolumn{6}{|c|}{$p=0.1$} \\
\hline \multicolumn{3}{|c|}{ Random Spin Configuration } & \multicolumn{3}{|c|}{ Good Spin Configuration } \\
\hline$<1 / s>_{u}$ & $u / U$ & $1+\frac{T^{U}-T^{D}}{T^{U}+T^{D}}$ & $<1 / s>_{u}$ & $u / U$ & $1+\frac{T^{U}-T^{D}}{T^{U}+T^{D}}$ \\
\hline $0.0049 \pm 0.0003$ & $0.53 \pm 0.13$ & $0.8 \pm 1.0$ & $0.0068 \pm 0.0005$ & $0.93 \pm 0.05$ & $1.8 \pm 0.6$ \\
\hline
\end{tabular}

We observe that the distribution of connectivity of the favoured spin type has shift to the left of the hampered spin's (Fig.1). This implies that the enhanced spin type should have more nodes with the same spin type in contact compared with the hampered one, a sign of the importance of local solidarity. 

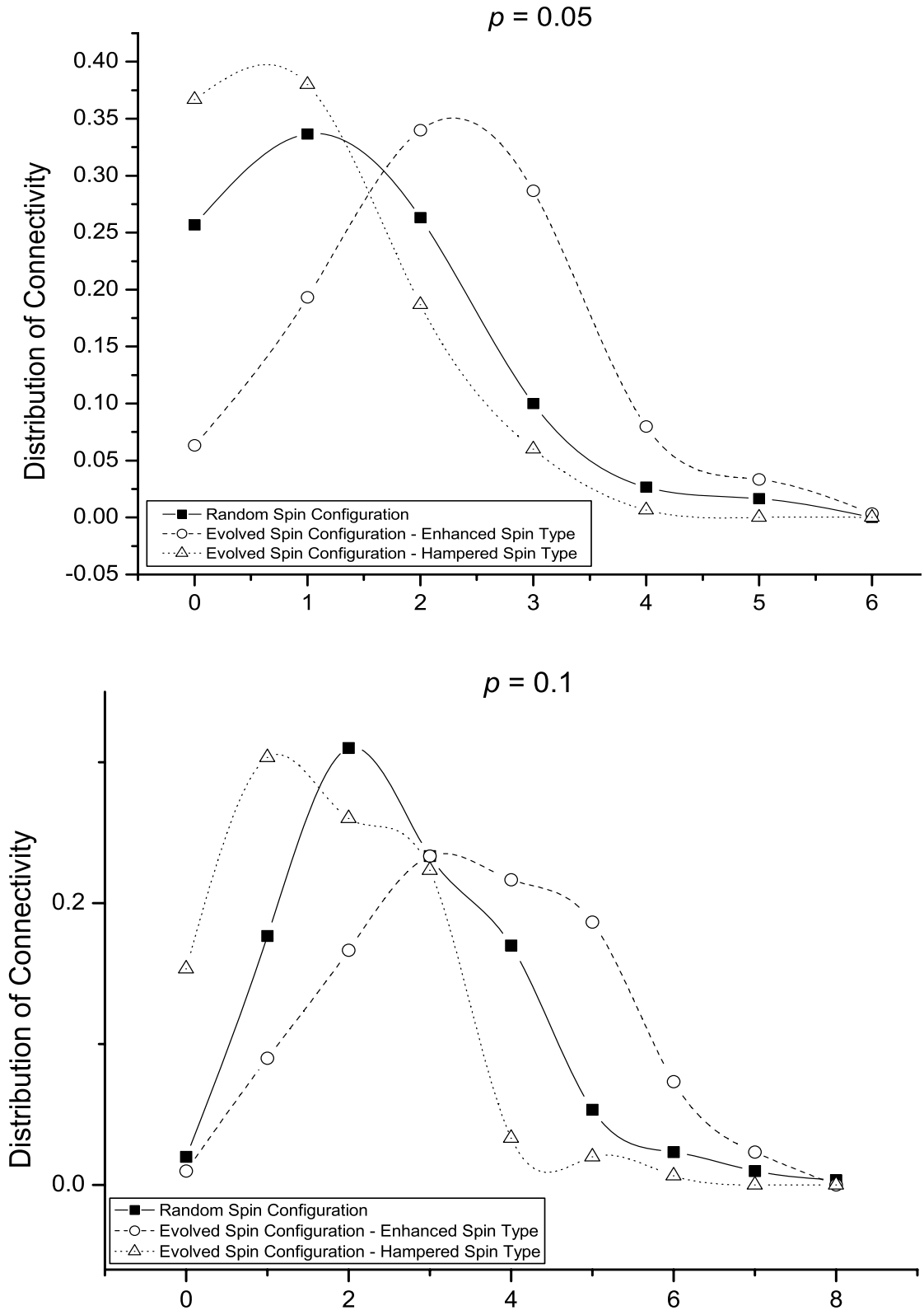

Fig. 1. Averaged Distribution of Connectivity $k_{i}$ for (1) spin up type in random configurations (Filled Square), (2) enhanced spin in evolved configurations (empty circle) and (3) hampered spin in evolved configurations (triangle) 
Based on numerical evidence, we conclude that the interconnectedness of nodes plays an important part in deciding which spin type to achieve dominance. In terms of the competing telecommunication service providers this means that if the clients of one service provider have a higher interconnectedness than the others' then it will have a higher probability to dominate the market. This agrees with our intuition on social behaviour: if a client has many friends and relatives subscribing the same cellular phone service provider, he/she is very unlikely to switch due to his or her friend's influence and the lower price on intra-network phone call. Thus, a company with limited resource should expand its market through existing clients, i.e. promote its service to the relatives, friends and acquaintances of the existing clients since this would enhance the interconnectedness among its client and they will tend to be loyal customers. This strategy to market dominance can also be viewed as a way to produce the maximum damage to other companies [10]. K.Y. Szeto acknowledges the support of CERG 6071-02P, 6157-01P, and DAG04/05SC23.

\section{References}

1. Kwok Yip Szeto and Chiwah Kong, Different Phases in a Supermarket Chain Network: An Application of an Ising Model on Soap Froth, Computational Economics, 22(2): 16317, 2003.

2. M. E. J. Newman, The Structure and Function of Complex Networks, SIAM Review 45, 167 (2003).

3. K.Huang, Statistical Mechanics, Wiley, 1987.

4. R. Albert and A.-L. Barabási, Statistical Mechanics of Complex Networks, Rev. Mod. Rev. 74, 47 (2002).

5. Xiujun Fu, Kwok Yip Szeto, and Wing Keung Cheung, Phase transition of twodimensional Ising model on random point patterns, Phys. Rev.E70, 056123(2004).

6. M. E. J. Newman and G. T. Barkema (1999), Monte Carlo Methods in Statistical Physics, Clarendon Press Oxford, 1999, p. 45-59

7. K.Y. Szeto and Zhang Jian, Adaptive Genetic Algorithm and Quasi-Parallel Genetic Algorithm: Application to Knapsack Problem (Lirkov, S. Marenov, and J. Wasniewski (eds.) June 2005, Sozopol, LSSC2005, LNCS 3743, pp.189-96, 2006,Springer-Verlag Berlin Heidelberg 2006).

8. Zhang Jian and K.Y. Szeto, Mutation Matrix in Evolutionary Computation: An Application to Resource Allocation Problem; Proceeding of the First International Conference on Natural Computation and The Second International Conference on Fuzzy Systems and Knowledge Discovery, 27-29 August 2005, Changsha, China, L. Wang, K. Chen, and Y.S. Ong (Eds.): ICNC 2005, LNCS 3612, pp. 112-119, 2005, Springer-Verlag Berlin Heidelberg 2005.

9. Chun Wai Ma and Kwok Yip Szeto, Locus Oriented Adaptive Genetic Algorithm: Application to the Zero/One Knapsack Problem, Proceeding of The 5th International Conference on Recent Advances in Soft Computing, RASC2004 Nottingham, UK. p.410415, 2004.

10. Z. Z. Guo, K.Y. Szeto, and Xiujun Fu, Damage spreading on two-dimensional trivalent structures with Glauber dynamics: Hierarchical and random lattices,Phys. Rev. E70, 016105(2004) 